\title{
Delivery of cisplatin and resiquimod in nanomicelles for the chemoimmunotherapy of ovarian cancer
}

\author{
Wen Yin ${ }^{*}$ and Sumin Qian
}

\section{*Correspondence:}

yinwenyu224911@163.com Gynecology Department II, Cangzhou Central Hospital, Cangzhou, Hebei, China

\begin{abstract}
Background: To explore the effect and mechanism of delivery of cisplatin (CDDP) and resiquimod in nanomicelles for the chemoimmunotherapy of ovarian cancer in vivo and in vitro.

Methods: Poly(L-glutamic acid)-graft-methoxypolyethylene glycols (PLG-g-mPEG) was used to carry cisplatin and resiquimod for the preparation of CDDP/resiquimod/ PLG-g-mPEG. We determined the loading content (LC) and encapsulation efficiency (EE), and then observed the particle shape, particle size distribution and zeta potential. In this study, we recruited 30 healthy adult participants and isolated mononuclear cells, and they were randomly classified into a control group, a CDDP group, a resiquimod group, a CDDP/resiquimod/PLG-g-mPEG group and a IFN- $\gamma+$ LPS group to identify macrophages markers in different polarization states. We conducted microprobe synchrotron radiation X-ray fluorescence (SRXRF) imaging to observe the cell uptake. Furthermore, we observed the effects of CDDP/resiquimod/PLG-g-mPEG on the growth and colony formation of SW626 cells and the expressions of apoptosis-associated genes and proteins. Tumor-bearing mouse models of ovarian cancer were prepared and randomized into a negative control group, a PLG-g-mPEG group, a CDDP group, a resiquimod group and a CDDP/resiquimod/PLG-g-mPEG group, so as to analyze the anti-cancer effect of CDDP/resiquimod/PLG-g-mPEG in vivo.

Results: The LC and EE of CDDP/resiquimod/PLG-g-mPEG were $19.42 \%$ and $90.12 \%$, respectively. Nanoparticles were uniform spherical in shape and closely arranged together, with a typical core-shell structure, and their average particle size and zeta potential were $82.36 \mathrm{~nm}$ and $-23.69 \mathrm{mV}$, respectively. When CDDP/resiquimod/PLGg-mPEG group was compared with the control group, the positive expression rate of CD16 in the CDDP/resiquimod/PLG-g-mPEG group highly increased, whereas the positive expression rate of CD163 dramatically decreased. In the meantime, Arg1 and Mrc1 mRNA expressions significantly decreased whereas IL-12 and NOS2 mRNA expressions dramatically increased $(P<0.05)$. Elemental mapping of cells exhibited notable internalization of cisplatin delivered by CDDP/resiquimod/PLG-g-mPEG to cytoplasm. We compared the cell survival rate between the CDDP/resiquimod/PLG-g-mPEG group and the control group, the CDDP/resiquimod/PLG-g-mPEG group sharply reduced
\end{abstract} author(s) and the source, provide a link to the Creative Commons licence, and indicate if changes were made. The images or other third party material in this article are included in the article's Creative Commons licence, unless indicated otherwise in a credit line to the material. If material is not included in the article's Creative Commons licence and your intended use is not permitted by statutory regulation or exceeds the permitted use, you will need to obtain permission directly from the copyright holder. To view a copy of this licence, visit http:// creativecommons.org/licenses/by/4.0/. The Creative Commons Public Domain Dedication waiver (http://creativecommons.org/publicdomain/zero/1.0/) applies to the data made available in this article, unless otherwise stated in a credit line to the data. 
$(P<0.05)$. What's more, the inhibitory effect got strengthened as the reaction time was prolonged, with the synergy coefficient of 0.31 .

Conclusion: PLG-g-mPEG-loaded CDDP and resiquimod effectively achieves the targeted delivery of chemotherapy and immunotherapy, with a strong synergistic anticancer effect.

Keywords: Ovarian cancer, Poly(L-glutamic acid)-graft-methoxypolyethylene glycols, Cisplatin, Resiquimod, M2 tumor-associated macrophages, Nanoparticles

\section{Background}

Ovarian cancer is one of the common cancers that adversely poses a threat to women's health, and its morbidity ranks third among female reproductive cancers, and its mortality ranks first among gynecological cancers (Luo et al. 2019). In general, patients with ovarian cancer have a poor prognosis with a 5-year survival rate of only 30 to $40 \%$ (Jin et al. 2017). Although surgery is the primary and most effective treatment for ovarian cancer, it almost does not work on patients with middle and advanced cancer and partial recurrence (Spiliotis et al. 2020). Therefore, in addition to surgery, chemotherapy is an important adjuvant treatment for patients with ovarian cancer. However, clinical practice has found that cisplatin (CDDP), a traditional chemotherapy drug, displays such limitations as low sensitivity, dose-dependence and cytotoxicity in the treatment of ovarian cancer (Le et al. 2017). Recent studies have demonstrated that the combination of immunotherapy with CDDP not only enhances the drug sensitivity of patients with ovarian cancer to a certain extent, but also increases the remission rate of cancers (Grabosch et al. 2019). Tumor-associated macrophages (TAMs) are immune cells that infiltrate tumor tissues. Recent studies have demonstrated that TAMs are differentiated into M1 phenotype and M2 phenotype due to the influence of tumor microenvironmental cytokines, with a high plasticity. M2-TAMs are abundantly present in majority of cancers to foster tumor growth.

Resiquimod (also named as R848) is a TLR7/8 agonist that belongs to the imidazoquinoline family (Thauvin et al. 2019; Li et al. 2021). Studies have revealed the anti-cancer effects of resiquimod. For example, resiquimod exhibits anti-tumor functions and attenuates cachexia in a murine model of pancreatic ductal adenocarcinoma (PDAC) (Michaelis et al. 2019). Resiquimod is posed as a potent inducer of cytokines in peripheral blood mononuclear cells and facilitates immune response (Thauvin et al. 2019). As an immune system activating agent, resiquimod was shown to drives M2 phenotype into M1 phenotype to inhibit tumor angiogenesis, and fully exerts its strong anti-cancer effect (Figueiredo et al. 2020). Resiquimod could enhance the immune response in mouse models of lymphoma, breast cancer, gastrointestinal tumor, head and neck cancer, and pancreatic cancer and so on (Scholch et al. 2015; Yin et al. 2015; O'Connor et al. 2017; Lu et al. 2019). However, the systematical injection and oral administration of resiquimod are severely affected by rapid plasma clearance and side effects including systemic inflammation and autoimmune responses (Pockros et al. 2007; Savage et al. 1996). On the other hand, the direct delivery of TLR agonists, including resiquimod, to tumors notably improves therapeutic efficacy on cancers (Rodell et al. 2018).

In recent years, nanoparticles have made great progress in the study of targeted tumor identification, of which, poly(L-glutamic acid)-graft-methoxypolyethylene glycols 
(PLG-g-mPEG) is a common passive targeted nanocarrier. Previous studies have shown that PLG-g-mPEG can carry two or more anti-cancer drugs with different mechanisms and exert a synergistic therapeutic effect ( $\mathrm{Li}$ et al. 2018). Yu and colleagues prepared a cisplatin-loaded PLG-g-mPEG nanoparticle via simple complexation of PLG-g-mPEG with cisplatin, and realized effective suppression of MCF-7 tumor growth (Yu et al. 2016). A multi-drug-loaded nanoparticle that contains resiquimod exhibited both chemo- and immune-therapy effect to ameliorate solid tumors resistant to first-line therapies (Silva et al. 2019). In view of the above, this study used PLG-g-mPEG to carry cisplatin and resiquimod to prepare CDDP/resiquimod/PLG-g-mPEG and explored its inhibitory impacts on macrophage polarization, tumor cell growth, aiming to offer more clinical references.

\section{Materials and methods}

Materials

Drugs

The nude mice were injected with cisplatin (Qilu(Hainan) Pharmaceutical Co., Ltd., National Medicine Standard: H20073653, specification $20 \mathrm{mg} /$ bottle), stored at $4{ }^{\circ} \mathrm{C}$ and protected from light. Resiquimod was purchased from Sigma company (USA) and its molecular mass was $314 \mathrm{Da}$. The nanocarrier materials of poly(L-glutamic acid)-graftmethoxypolyethylene glycols (PLG-g-mPEG) were provided by Changchun Institute of Applied Chemistry, Chinese Academy of Sciences.

\section{Cells}

SW626 cells were human ovarian adenocarcinoma cells purchased from Ningbo Mingzhou Biotechnology Co., Ltd. (Product No.: MZ-1397). Firstly, we took a sterile tube consisting of $1 \mathrm{~mL}$ of cell suspension and shook it immediately at $37{ }^{\circ} \mathrm{C}$ water bath to thaw. Secondly, we added $4 \mathrm{~mL}$ of culture medium and then centrifuged it at $1000 \mathrm{r} / \mathrm{min}$ for $4 \mathrm{~min}$ and discarded the supernatant. Next, we added $1 \mathrm{~mL}$ of culture medium, blew well and placed it in a $37{ }^{\circ} \mathrm{C}$ and $5 \% \mathrm{CO}_{2}$ culture flask. When the cell density was up to 80 to $90 \%$, we carried out subculture. Then we rinsed cells one or two times with phosphate-buffered saline (PBS), with 5 min each time. Next, we dripped $2 \mathrm{~mL}$ of trypsin to digest in a $37{ }^{\circ} \mathrm{C}$ incubator for one or two minutes. Next, we observed the cell morphology under a microscope. When most of cells became round and fell off, we added a small amount of culture medium to stop the digestion. $6-8 \mathrm{~mL}$ of medium was supplemented per tube, and then we gently mixed and aspirated the solution. Next, we centrifuged it at $1000 \mathrm{r} / \mathrm{min}$ for $4 \mathrm{~min}$, discarded the supernatant, supplemented the medium and blew the solution well. In the end, we divided the cell suspension into a culture flask comprising $8 \mathrm{~mL}$ of culture medium with a ratio between $1: 2$ and $1: 5$, and then cultured at $37^{\circ} \mathrm{C}$ and $5 \% \mathrm{CO}_{2}$.

\section{Main reagents}

Trypsin solution was purchased from Suzhou New Semi Biotech Co., Ltd (Product No.: C125C1). Lymphocyte separation medium was purchased from Shanghai Meijing Biotechnology Co., Ltd (Product No.: IAE-1). Hanks solution was purchased from Wuhan Purity Biotechnology Co., Ltd (Product No.: CDLG-3745). FITC-labeled 
mouse anti-human CD14 antibody (Product No. GH0573), Trizol reagent (Product No. GL1311) and Super Sensitive ECL Chemiluminescent Kit (Product No. YT061) were purchased from Beijing Biolab Technology Co., Ltd. RPMI-1640 culture medium (Product No.: PM150110B) was purchased from Wuhan Punuosai Life Technology Co., Ltd. PMA solution (Product No.: P6741), MTT reagent (Product No.: M8180), cell total protein extraction kit (Product No.: BC3790) and BCA protein concentration determination kit (Product No.: PC0020) were purchased from Beijing Soleibao Biotechnology Co., Ltd. Human recombinant macrophage colony stimulating factor (M-CSF, Product No. CYT-308) was purchased from Wuhan Aimeijie Technology Co., Ltd. Reverse transcription kit was purchased from Beijing Zhijie Fangyuan Technology Co., Ltd (Product No.: K1622). Mouse anti-Bak monoclonal antibody (Product No.: ab104124), rabbit anti-Bid monoclonal antibody (Product No.: ab32060), rabbit anti-Bim monoclonal antibody (Product No.: ab32158), rabbit anti-BCL-2 monoclonal antibody (Product No.: ab182858), rabbit anti-Caspase-3 monoclonal antibody (Product No.: ab32351), rabbit anti-Caspase- 9 monoclonal antibody (Product No.: ab32539), mouse anti- $\beta$-actin monoclonal antibody (Product No.: ab6276) and HRP-labeled rabbit anti-mouse IgG antibody (Product No.: ab6728) were purchased from Abcam company, USA.

\section{Instruments}

Transmission electron microscope (Model: TALOSL120CMS), flow cytometry (Model: Attune NxT) and chemiluminescence imaging system (Model: iBright) were purchased from Thermo Fisher Company, USA. Dynamic light scattering laser particle size analyser (Model: Mastersizer 3000E) was purchased from Malvern PANalytical Instruments, UK. RT-PCR instrument was purchased from Roche, Switzerland (Model: Light Cycler96). Confocal laser microscopy was purchased from Keyence Corporation, Japan (Model: VK-9700).

\section{Preparation of CDDP/resiquimod/PLG-g-mPEG (Meiling et al. 2019)}

Firstly, we weighed $400 \mathrm{mg}$ of CDDP and $200 \mathrm{mg}$ of resiquimod and then dissolved them in $150 \mathrm{~mL}$ of ultrapure water. Next, the mixture was shook evenly for $24 \mathrm{~h}$ at $37^{\circ} \mathrm{C}$ in the dark, and we labeled it as solution A. We weighed $1.6 \mathrm{~g}$ of PLG-g-mPEG and dissolved them in $50 \mathrm{~mL}$ ultrapure water. Next, the mixture was stirred in a magnetic stirrer at $37^{\circ} \mathrm{C}$ for $24 \mathrm{~h}$ at a constant speed, and then we labeled it as solution B. After $24 \mathrm{~h}$, we observed whether the solution A was thoroughly dissolved or not. If yes, we mixed solution $\mathrm{A}$ and solution $\mathrm{B}$, and the mixture was shook evenly for $72 \mathrm{~h}$ at $37^{\circ} \mathrm{C}$ in the dark, and then we labeled it as solution C. We poured distilled water into a clean beaker and boiled it, and then poured it in a dialysis bag (molecular weight: 5000), lasting 5 min of boil, and finally we carried out activation. Next, we put the activated dialysis bag into a clean beaker consisting of ultrapure water to rinse its inside. Next, we poured solution $\mathrm{C}$ with $72 \mathrm{~h}$ of reaction into the activated dialysis bag, used deionized water to conduct dialysis and replaced the dialysate every $4 \mathrm{~h}$, which lasted at least $24 \mathrm{~h}$ in the dark. We collected the solution in the dialysis bag and freeze-dried it in the dark to obtain CDDP/ resiquimod/PLG-g-mPEG powder, and then sealed and stored this powder at $-20{ }^{\circ} \mathrm{C}$ in the dark. The dose of CDDP/resiquimod/PLG-g-mPEG used for in vitro experiments was $50 \mu \mathrm{g} / \mathrm{mL}$. 


\section{Determination of loading content (LC) and encapsulation efficiency (EE)}

$\mathrm{LC}(\%)=($ cisplatin mass in CDDP/resiquimod/PLG-g-mPEG)/CDDP/resiquimod/PLGg-mPEG mass $\times 100 \%, E E(\%)=($ cisplatin mass in CDDP/resiquimod/PLG-g-mPEG) $/$ (cisplatin mass added in the reaction system) $\times 100 \%$. We repeatedly carried out this experiment for 3 times and then took the average value. The ideal standard of nanodrugs was $\mathrm{EE} \geq 90 \%$.

\section{Morphology, particle size distribution and zeta potential}

(1) Transmission electron microscope: $5 \mathrm{mg}$ of CDDP/resiquimod/PLG-g-mPEG powder was dissolved in $5 \mathrm{~mL}$ of ultrapure water, and the mixture was diluted at $1 \mathrm{mg} /$ $\mathrm{mL}$ concentration. Next, we collected $1 \mathrm{~mL}$ of solution and dripped it onto a carbon film supported by 500 mesh copper grid. Next, the solution was dried naturally in a dust-free environment, and we observed the morphology of nanoparticles on the carbon film under a transmission electron microscope.

(2) Particle size distribution and zeta potential: $5 \mathrm{mg}$ of CDDP/resiquimod/PLGg-mPEG powder was dissolved in $5 \mathrm{~mL}$ of ultrapure water, and the mixture was placed in a cuvette, and a nanoparticle tracking analysis (NTA) (Nanosight LM10, Malvern, UK) was applied to determine the particle size distribution of the nanodrugs and zeta potential. The test cycle numbers were automatically set by the detection system according to the varied sample concentration, which ranged from 3 to 13 cycles in our tests. The assay was repeatedly performed three times on samples in each group, and then we took the average value, and the ideal standard particle size of nano-drugs ranged from $5 \mathrm{~m}$ to $200 \mathrm{~nm}$.

(3) Fourier transform infrared spectroscopy (FTIR) analysis: the functional groups of the PEG-COOH, cisplatin, and resiquimod were examined by FTIR, respectively. Samples were freeze-dried, mixed with $\mathrm{KBr}$, pressed into a disk, and scanned from 500 to $4000 \mathrm{~cm}^{-1}$.

\section{In vitro experiments}

\section{Mononuclear cell isolation}

Density gradient centrifugation was applied. Firstly, the peripheral venous blood samples were collected from 30 healthy adult participants in this study. Next, a sample was placed in an anticoagulant tube consisting of EDTA, and then diluted with an equal volume of PBS. Next, we slowly added the lymphocyte separation solution at a ratio of 2:1, and centrifuged it at $2000 \mathrm{r} / \mathrm{min}$ for $20 \mathrm{~min}$. Thereafter we used a pipette to aspirate the tunica albuginea layer between the upper layer and the middle layer, and performed further centrifugation at $800 \mathrm{r} / \mathrm{min}$ for $5 \mathrm{~min}$. Next, we discarded the supernatant and rinsed it with PBS twice, 5 min each time, and added Hanks solution to digest the cells, followed to beat into a single cell suspension with a pipettor. Next, we resuspended the cells in PBS, added FITC-labeled mouse anti-human CD14 antibody, and they were cultured at $4^{\circ} \mathrm{C}$ for $30 \mathrm{~min}$. Next, we rinsed them with pre-cooled PBS for 3 times, $5 \mathrm{~min}$ each time, centrifuged at $2000 \mathrm{r} / \mathrm{min}$ for $20 \mathrm{~min}$ and discarded the supernatant. We 
determined the CD14-positive expression rate on the cell surface via flow cytometry, screened out cells with a positive rate of $90 \%$ or more, and stored them at $-80^{\circ} \mathrm{C}$ for preparation. All experiments were conducted under the approval of Ethic Committee of Cangzhou Central Hospital. Informed consents were acquired from all participants before the experiments.

\section{Macrophages-induced formation}

Firstly, we inoculated human mononuclear cells in a 6-well plate, and added RPMI1640 medium to dilute the cell density to $2 \times 10^{5}$ cells $/ \mathrm{mL}$, and then inoculated in a 24-well plate at $200 \mu \mathrm{L} /$ well. Next $100 \mathrm{ng} / \mathrm{mL}$ of PMA was added for reaction at $37{ }^{\circ} \mathrm{C}$ for $48 \mathrm{~h}$, and we observed the cell growth under an optical microscope, and collected adherent growth cells. Samples were randomized into a control group, a CDDP group, a resiquimod group, a CDDP/resiquimod/PLG-g-mPEG group and an IFN- $\gamma+$ LPS group. The IFN- $\gamma+$ LPS group represented the positive control group, the induction was continued with $10 \mathrm{ng} / \mathrm{mL}$ M-CSF for 6 days, and the medium was changed half a day. DMEM/F-12+10\% FBS $+100 \mathrm{ng} / \mathrm{mL}$ LPS $+20 \mathrm{ng} / \mathrm{mL}$ IFN- $\gamma$ and DMEM/F-12 + 10\%FBS $+10 \mathrm{ng} / \mathrm{mL}$ IL-4 were correspondingly added in each group and cultured at $37^{\circ} \mathrm{C}$ for $24 \mathrm{~h}$ to classify macrophages in different polarization states.

\section{Identification of $M 1$ and $M 2$ macrophage markers}

(1) Flow cytometry: M2-macrophages were induced with resiquimod, CDDP/resiquimod/PLG-g-mPEG for 3 days, $50 \mathrm{ng} / \mathrm{mL}$ IFN- $\gamma+10 \mathrm{ng} / \mathrm{mL}$ LPS as the positive control group. Flow cytometry was used to detect the positive expression rates of CD16, a surface marker of M1 macrophages and CD163, a surface marker of M2-macrophages. (2) RT-PCR: Trizol reagent was used to extract total cell RNA while UV-vis spectrophotometer used for quantitative and purity analysis. Absorbance (A) was determined at $260 \mathrm{~nm}$ and $280 \mathrm{~nm}$, and the ratio of A260nm/A280nm ranged from 1.8 to 2.0. RNA was stored at $-80^{\circ} \mathrm{C}$ for preparation, and we followed the kit manual to transfer the reverse transcription of RNA into cDNA. IL-12 and NOS2 were known as M1-type macrophage markers while Arg1 and Mrc1 as M2-type macrophage markers. Sangon (Shanghai) Biotechnology Company designed and synthesized primers that were as follows: IL-12 upstream primer $5^{\prime}$-CCACTACACCTGTCACAAAGG-3', downstream primer $5^{\prime}$-TTG AGCTTGTGAACGGCATCC-3', NOS2 upstream primer 5'-AAGGTGTGCCACGTT CCATG-3', downstream primer 5'-AAGTGGCTAG'CCATGCATG-3, Arg1 upstream primer 5'-GCGGAATTCTTAAAGAAG-3', downstream primer 5'-AGTCTTTACTGT ACCTGAACAG-3', Mrc1 upstream primer 5'-TAGCCAAGGGTACCACAG-3', downstream primer $5^{\prime}$-GGTTAACGTGTTCCAAGTC-3', $\beta$-actin upstream primer $5^{\prime} \mathrm{CAC}$ AGGTCAAGTC-3' ${ }^{\prime}$, downstream primer $5^{\prime}$-GCACGAAGGCTCATGGTTCA-3'. Individual $20 \mu \mathrm{L}$ PCR reaction system consisted of SYBR ${ }^{\circledR}$ Green $10 \mu \mathrm{L}$, cDNA $1 \mu \mathrm{L}$, RNasefree Water $7 \mu \mathrm{L}$, upstream primer $1 \mu \mathrm{L}$ and downstream primer $1 \mu \mathrm{L}$. Reaction program: the initial step was to perform once pre-denaturation at $95^{\circ} \mathrm{C}$ for $10 \mathrm{~min}$, and the second step was denaturation at $95^{\circ} \mathrm{C}$ for $10 \mathrm{~s}$. in the third step, the temperature was reduced to $60{ }^{\circ} \mathrm{C}$ so that the primer could anneal and extend for $30 \mathrm{~s}$. In general, 45 cycles produced a sufficient amount of RNA. RT-PCR instrument was used for quantitative analysis in conjunction with software Light Cycler96. 


\section{Microprobe synchrotron radiation X-ray fluorescence (SRXRF) imaging}

The uptake of CDDP/resiquimod/PLG-g-mPEG by cells was determined by microprobe SRXRF analysis following a previously reported protocol (Davis et al. 2012). In brief, the SW626 cells were treated and collected as cell pellet, fixed, dehydrated with ethanol and Spurrs. The cells were then transferred to a Beem capsule, centrifuged to form a pellet, cured at $60^{\circ} \mathrm{C}$ for $12 \mathrm{~h}$, followed by sectioning to $1 \mu \mathrm{m}$ slices. The slices were then dyed with toluidine blue and mounted onto gold Finder grids. X-rays were detected in the fluorescence mode.

\section{The effect of CDDP/resiquimod/PLG-g-mPEG on SW626 cells growth}

MTT assay was applied. We diluted SW626 cells with trypsin, and inoculated them in 96-well plates at $1 \times 10^{5}$ cells/well. They were randomized into a negative control group, a PLG-g-mPEG group, a CDDP group, a resiquimod group and a CDDP/ resiquimod/PLG-g-mPEG group, and placed in a $37{ }^{\circ} \mathrm{C}, 5 \% \mathrm{CO} 2$ incubator overnight for incubation. PLG-g-mPEG $(50 \mu \mathrm{g} / \mathrm{mL})$, CDDP $(2 \mu \mathrm{g} / \mathrm{mL})$, resiquimod $(2 \mu \mathrm{g} / \mathrm{mL})$ and CDDP/resiquimod/PLG-g-mPEG $(50 \mu \mathrm{g} / \mathrm{mL})$ were, respectively, added in the PLG-g-mPEG group, the CDDP group, the resiquimod group and the CDDP/resiquimod/PLG-g-mPEG group, and an equal volume of DMEM was added into the negative control group. Next $100 \mu \mathrm{L}$ of MTT reagent was added into each group after $24 \mathrm{~h}$, $48 \mathrm{~h}, 72 \mathrm{~h}$ incubation, respectively, and they were incubated at $37^{\circ} \mathrm{C}$ for $4 \mathrm{~h}$. Next, we discarded the supernatant, added $150 \mathrm{~mL}$ of DMSO, and oscillated it at $37{ }^{\circ} \mathrm{C}$ for $10 \mathrm{~min}$. A value was at the wavelength of $490 \mathrm{~nm}$ measured by the microplate reader. Cell survival rate $(\%)=\mathrm{A} 490 \mathrm{~nm}$ in experimental groups/A490 $\mathrm{nm}$ in the control group $\times 100 \%$. Median inhibitory concentration (IC50) was the drug concentration when the cell survival rate was $50 \%$, and the combination index $(\mathrm{CI})=\mathrm{D} 1 / \mathrm{Df} 1+\mathrm{D} 2 /$ Df2. D1 and D2 expressed the drug concentration when drugs were jointly used and the cell survival rate was $50 \%$, and Df1 and Df2 expressed the drug concentrations when drugs were used alone and the cell survival rate was $50 \%$. CI $>1$ indicated antagonism, $\mathrm{CI}=1$ indicated additive effect, $\mathrm{CI} \geq 0.8$ indicated low synergy, $0.6 \leq \mathrm{CI}<0.8$ indicated moderate synergy, and $0.2 \leq \mathrm{CI}<0.4$ indicated strong synergy.

\section{The effect of CDDP/resiquimod/PLG-g-mPEG on the clonogenic ability of SW626 cells}

Plate cloning assay was performed (Franken et al. 2006). Firstly, we diluted SW626 cells with trypsin to prepare cell suspension. Next, they were inoculated with 100 cells/well in a 24 -well plate consisting of $10 \%$ fetal bovine serum, and cultured at $37^{\circ} \mathrm{C}$ and $5 \% \mathrm{CO}_{2}$ for $2-3$ weeks. When seeing clones in the petri dish with naked eye, we discarded the supernatant and soaked the cells into PBS twice to terminate the culture. Next, we added methanol to stabilize the state for $15 \mathrm{~min}$, and then dripped Giemsa stain for 10-30 min. Next, we rinsed them slowly with running water and dried naturally, and then placed the petri dish upside down on the transparent film with the grid. In the end, we counted clones composing of over 50 cells under the microscope. The clone formation rate (\%) was calculated by the ratio of colony number to seeded cell number. 


\section{Apoptosis-associated gene and protein expression}

We used western blotting assay. Firstly, we diluted SW626 cells with trypsin, and inoculated them in a 6-well plate $1 \times 1$ with $0^{6}$ cells/well, and the cell processing was the same as 1.5.5. After $48 \mathrm{~h}$, we followed the kit manual to extract cell total protein using column method. BCA was applied to detect protein concentration and stored at $-80{ }^{\circ} \mathrm{C}$ for preparation. Separation gel and concentrated gel were required for preparation of SDS-polyacrylamide gel electrophoresis (SDS-PAGE). Then we took protein sample for electrophoresis, set separation gel at $120 \mathrm{~V}$ and concentrated gel at $80 \mathrm{~V}$, with two or three hours of electrophoresis. After the electrophoresis ran, we carried out wet transfer to NC membrane, and 5\% skimmed milk powder was sealed and stored at room temperature for $2 \mathrm{~h}$. Then we dripped mouse anti-Bak monoclonal antibody, rabbit anti-Bid monoclonal antibody, rabbit anti-Bim monoclonal antibody, rabbit anti-BCL-2 monoclonal antibody, rabbit anti-Caspase-3 monoclonal antibody, rabbit anti-Caspase-9 monoclonal antibody, rabbit anti-Arg1 monoclonal antibody, rabbit anti-Mrc1 monoclonal antibody, rabbit anti-IL-12 monoclonal antibody, rabbit anti-NOS2 monoclonal antibody, and $\beta$-actin was used for internal control overnight at $4{ }^{\circ} \mathrm{C}$. The membrane was rinsed with Tris-buffered saline with Tween 20 (TBST), and then we added secondary antibody, and incubated the membrane at room temperature for $1 \mathrm{~h}$. Next, we rinsed the membrane with TBST and followed the kit manual to get color development using ECL and exposure using chemiluminescence imaging system.

\section{In vivo experiment}

A total of 50 female BALB/c-Nude nude mice with SPF grade were purchased from Changzhou Cavens Experimental Animal Co., Ltd., aging from 4 to 6 weeks old, and the experimental animal license number was SCXK (Su) 2016-0010. They were equally randomized into a negative control group, a PLG-g-mPEG group, a CDDP group, a resiquimod group and a CDDP/resiquimod/PLG-g-mPEG group. They were raised at between 20 and $26^{\circ} \mathrm{C}$, with the relative humidity of $40-70 \%$ and the light of $12 / 12 \mathrm{~h}$, and freely ate and exercised. The animal study was reviewed and approved by the Ethic Committee of Cangzhou Central Hospital. The experiments were performed in strict accordance to the Guide for the Care and Use of Laboratory animals published by the US National Institutes of Health. We diluted SW626 cells with trypsin and modified the cell concentration to $1-2 \times 10^{7}$ cells $/ \mathrm{mL}$. Mice were subcutaneously inoculated $0.2 \mathrm{~mL}$ of SW626 cells in the right axilla, and we measured the maximum diameter of tumor tissue. When the maximum diameter reached $1.0 \mathrm{~cm}$, PLG-gmPEG, CDDP, resiquimod and CDDP/resiquimod/PLG-g-mPEG were, respectively, injected at caudal vein, and the dose was controlled at the amount that did not cause death or $20 \%$ or more weight loss in mice. Then the negative control group was given an equal volume of PBS, with 2 times per week. Mouse body weight and tumor weight were recorded every 2 weeks. Tumor inhibition rate $(\%)=(1-$ tumor weight in the treatment group/tumor weight in the control group) $\times 100 \%$. The length and width of tumors were recorded every 3 days (same time as the recording of body weight), and tumor volume was calculated by the following formula: length $(\mathrm{mm}) \times$ width $(\mathrm{mm})^{2} / 2$. 


\section{Statistical analysis}

For all the data in this study, statistical analysis was performed using SPSS20.0 software. The measurement data were described as $( \pm s)$, and one-way analysis of variance was applied for the comparison among multiple groups, and the Snk-q test was used for comparison between groups. The count data were described as frequency or composition ratio with the $\chi^{2}$ test. $P$-values $\leq 0.05$ were considered to be significant.

\section{Results}

\section{Physicochemical characterization of CDDP/resiquimod/PLG-g-mPEG}

A standard curve was drawn with different concentrated CDDP solutions. Then the CDDP/resiquimod/PLG-g-mPEG was dissolved in water. The absorbance of CDDP encapsulated in the nanomicelles was measured by UV spectrophotometer at $705 \mathrm{~nm}$, followed by calculation by using the standard curve. The obtained mass of CDDP was then put in formulas as following: $\mathrm{LC}(\%)=(\mathrm{CDDP}$ mass in $\mathrm{CDDP} / \mathrm{resiquimod} /$ PLG-g-mPEG)/CDDP/resiquimod/PLG-g-mPEG mass $\times 100 \%, \quad$ EE $\quad(\%)=($ CDDP mass in CDDP/resiquimod/PLG-g-mPEG)/(cisplatin mass added in the reaction system) $\times 100 \%$. The LC and EE of CDDP/resiquimod/PLG-g-mPEG were $19.42 \%$ and $90.12 \%$, respectively. The structure of resiquimod is shown in Fig. 1A. Nanoparticles were uniform spherical in shape and closely arranged together, with a typical core-shell structure (Fig. 1B), and their average particle size and zeta potential were $82.36 \mathrm{~nm}$ (Fig. 1C) and $-23.69 \mathrm{mV}$ (Fig. 1D), respectively. Figure 1E shows the characterized absorption peaks of CDDP, resiquimod, and PLG-g-mPEG at $1317 \mathrm{~cm}^{-1}$, $3275 \mathrm{~cm}^{-1}, 1630 \mathrm{~cm}^{-1}, 1527 \mathrm{~cm}^{-1}$, and $1453 \mathrm{~cm}^{-1}$.

\section{Identification of $\mathrm{M} 1$ and $\mathrm{M} 2$ macrophage markers}

Flow cytometry revealed that when CDDP/resiquimod/PLG-g-mPEG group was compared with the control group, the positive expression rate of CD16 in the CDDP/ resiquimod/PLG-g-mPEG group highly increased, whereas the positive expression rate of CD163 dramatically decreased $(P<0.05)$. In the meantime, Arg1 and Mrc1 mRNA expressions in the CDDP/R848/PLG-g-mPEG group significantly decreased whereas IL-12 and NOS2 mRNA expressions dramatically increased $(P<0.05)$; see Table 1 and Fig. 2A. The results from western blotting further conformed the altered protein levels of Arg1, Mrc1, IL-12 and NOS2 (Fig. 2B).

\section{The effect of CDDP/resiquimod/PLG-g-mPEG on SW626 cells uptake}

We examined the internalization of nanomicelles into the SW626 cells by microprobe SRXRF imaging. The microprobe SRXRF imaging could detect metal or other selected elements, which in our case is cisplatin, in cells with a super high sensitivity (submicron resolution), and do not need radioactive or fluorescent labeling. The SRXRF provided elemental mapping to determine the amount of cisplatin that entered cells. As shown by elemental maps in Fig. 3, the maximum cisplatin concentration of $0.0707 \mu \mathrm{g} / \mathrm{cm}^{2}$ in CDDP/resiquimod/PLG-g-mPEG-treated cells was remarkably 


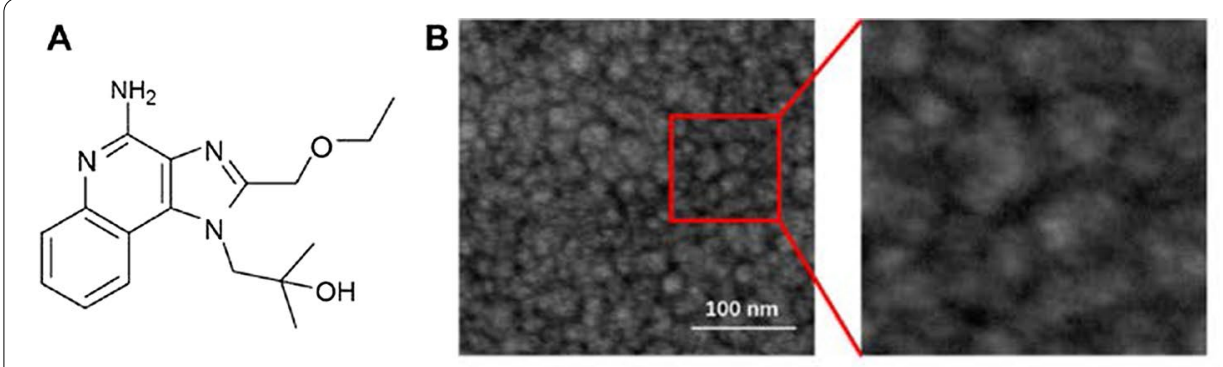

C

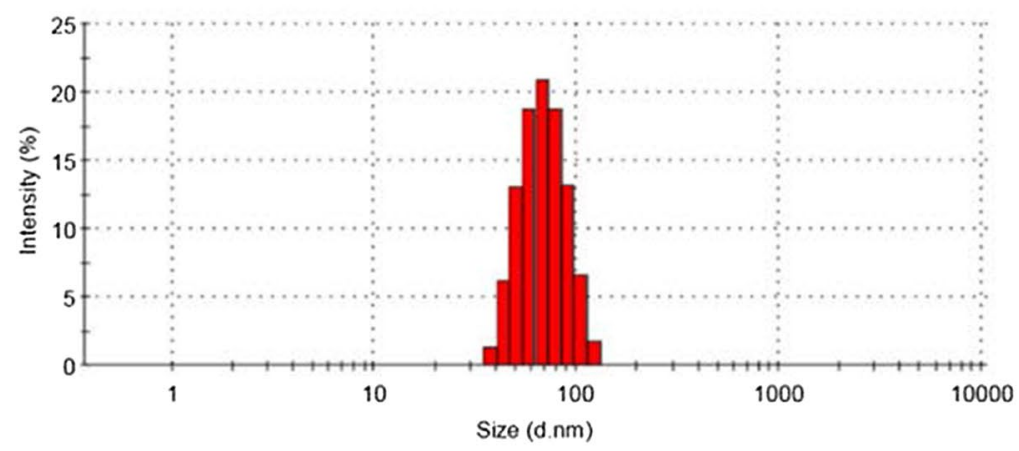

Zeta Potential Distribution

D

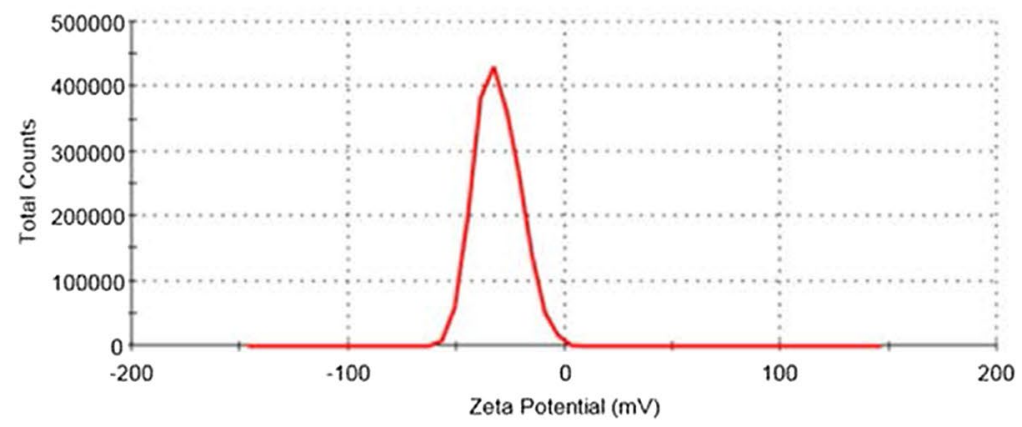

$\mathbf{E}$

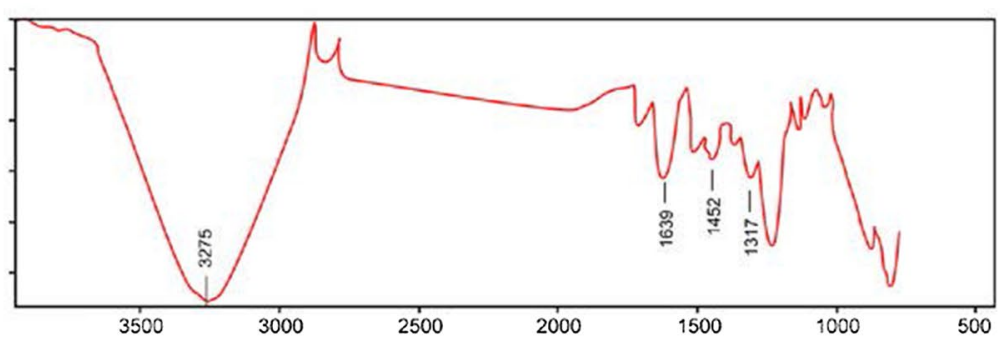

Fig. 1 Physicochemical characterization of CDDP/resiquimod/PLG-g-mPEG. A for structure of resiquimod. B for morphology; C for distribution of particle size; $\mathbf{D}$ for zeta potential, $\mathbf{E}$ for FTIR analysis

higher than that of untreated control cells $\left(0.0139 \mu \mathrm{g} / \mathrm{cm}^{2}\right)$. These results suggested the effective uptake of CDDP/resiquimod/PLG-g-mPEG by tumor cells.

\section{The effect of CDDP/resiquimod/PLG-g-mPEG on the growth of SW626 cells}

Compared against the control group, PLG-g-mPEG almost did not influence the growth of SW626 cells, and as the reaction time was prolonged, the cell survival rate 
Table 1 Identification of M1 and M2 macrophage markers with flow cytometry

\begin{tabular}{lll}
\hline Group & CD16-positive expression rate & $\begin{array}{l}\text { CD163-positive } \\
\text { expression rate }\end{array}$ \\
\hline Control group & $15 \%$ & $72 \%$ \\
CDDP & $16 \%$ & $70 \%$ \\
Resiquimod & $35 \%$ & $19 \%$ \\
IFN- + LPS & $48 \%$ & $25 \%$ \\
CDDP/resiquimod/PLG-g-mPEG & $65 \%{ }^{(1)}$ & $15 \%$ (1)(2)(3) \\
\hline
\end{tabular}

(1) $P<0.05$ means the comparison with the control group

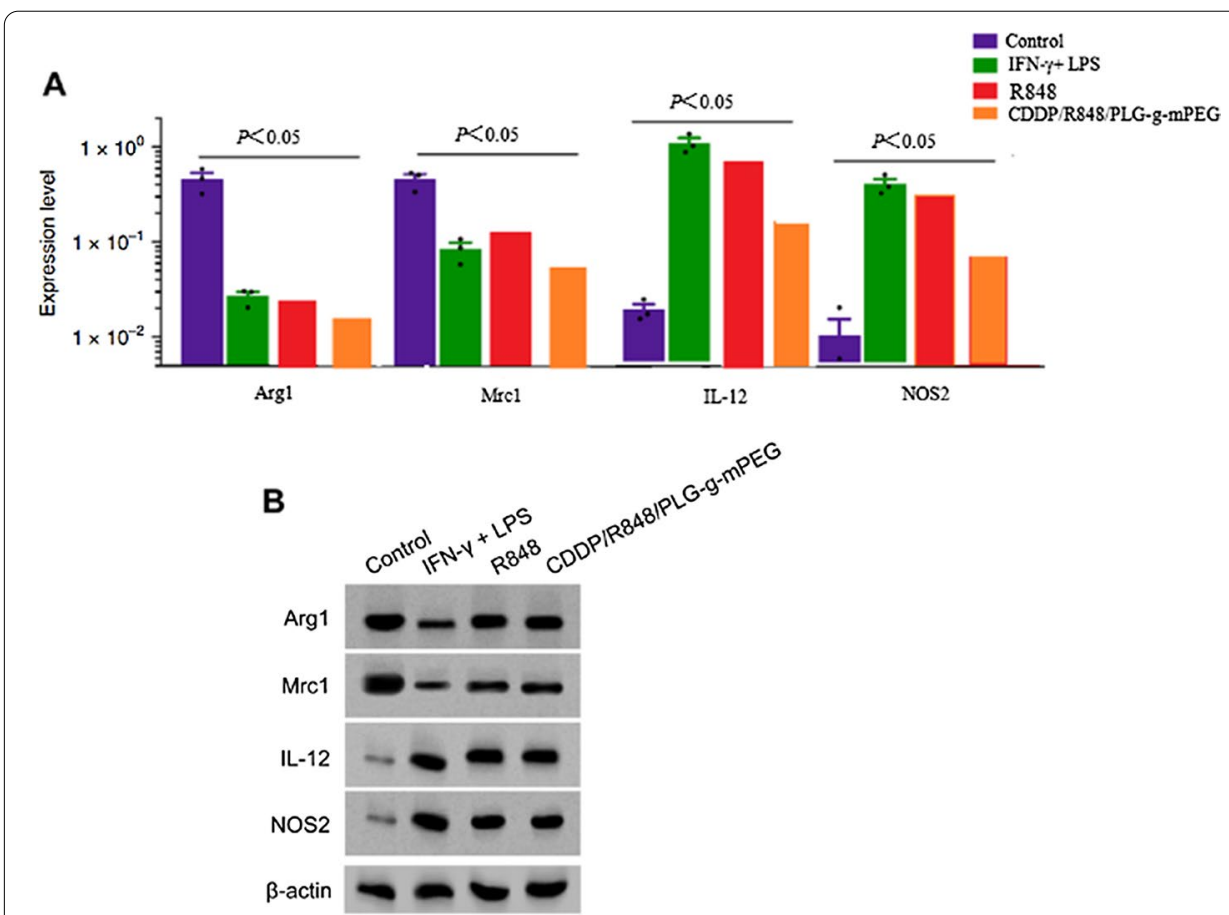

Fig. 2 Identification of $\mathrm{M} 1$ and $M 2$ macrophage markers with RT-PCR and western blotting

was maintained above $90 \%$. We compared the cell survival rate between the control group and the CDDP/resiquimod/PLG-g-mPEG group, and the CDDP/resiquimod/ PLG-g-mPEG significantly reduced $(P<0.05)$, and as the reaction time was prolonged, the inhibitory effect of CDDP/resiquimod/PLG-g-mPEG on the growth of SW626 cells was increasingly enhanced, with CI of 0.31 (see Fig. 4).

The effect of CDDP/resiquimod/PLG-g-mPEG on the clonogenic ability of SW626 cells

We compared the plate colony formation rate between the control group and the CDDP/resiquimod/PLG-g-mPEG group, and the CDDP/resiquimod/PLG-g-mPEG group sharply reduced $(P<0.05)$, and as the reaction time was prolonged, the inhibition of CDDP/resiquimod/PLG-g-mPEG in the colony formation of SW626 cells got enhanced (see Fig. 5). 

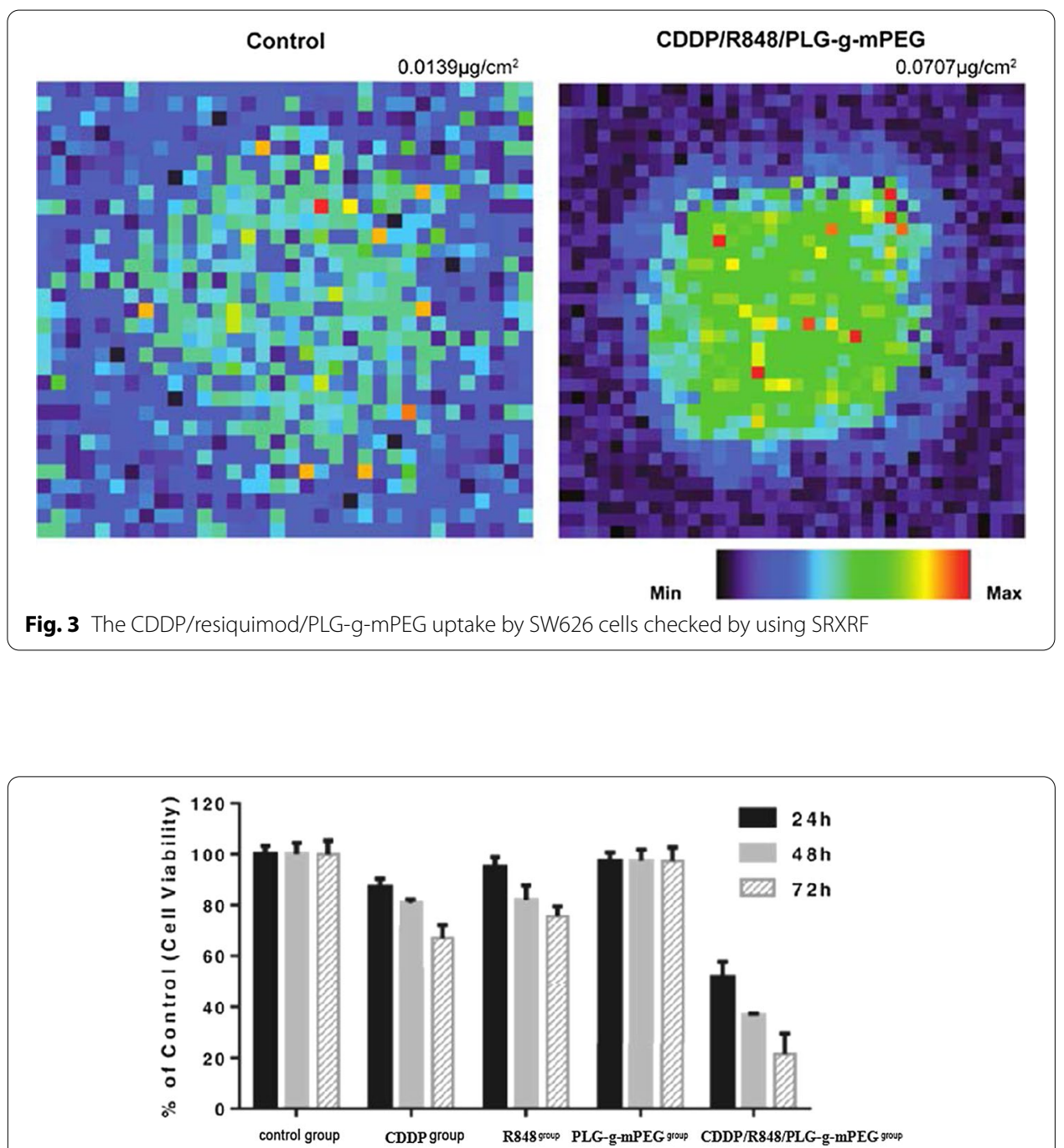

Fig. 4 The effect of CDDP/resiquimod/PLG-g-mPEG on the growth of SW626 cells

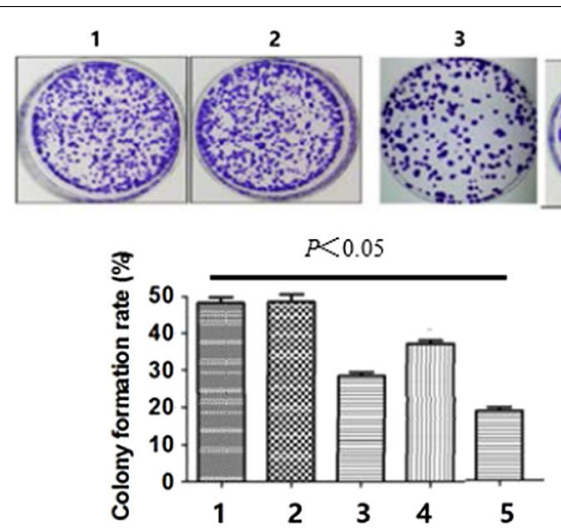

Fig. 5 The effect of CDDP/resiquimod/PLG-g-mPEG on clonogenic ability of SW626 cells. 1 for control group; 2 for CDDP group; 3 for resiquimod group; 4 for PLG-g-mPEG group; 5 for CDDP/resiquimod/PLG-g-mPEG group 
The effect of CDDP/resiquimod/PLG-g-mPEG on the expressions of apoptosis-associated genes and proteins in SW626 cells

Compared against the control group, there was sharp increase in the relative expressions of Bak, Bid, Bim, Caspase-3 and Caspase-9 in the CDDP/resiquimod/PLG-g-mPEG group, whereas the relative expression of BCL-2 decreased significantly $(P<0.05)$; see Fig. 6.

\section{The inhibitory effect of CDDP/resiquimod/PLG-g-mPEG on cancers in the body}

The weight of mice in each group fluctuated during treatment, but there was no $\geq 20 \%$ weight loss or death (Fig. 7A). Compared against the negative control group, resiquimod did not work on cancers, but CDDP, PLG-g-mPEG and CDDP/resiquimod/PLGg-mPEG dramatically inhibited the growth of tumor cells, and CDDP/resiquimod/ PLG-g-mPEG was the most effective drug for the inhibition of cancers $(P<0.05)$, as manifested by the tumor weight and tumor growth curve (Fig. 7B, C).

\section{Discussion}

Poor targeting is one of the leading causes of the side effects of chemotherapy drugs. For instance, it has strong harm on normal cells and tissues simultaneously while killing tumor cells (Abouaitah et al. 2020). The emergence of nanocarriers enables the distribution of chemotherapeutic drugs in the body to be more reasonable, which not only effectively enhances the inhibition of drugs on tumor cells, but also alleviates adverse reactions and assists patients in overcoming the resistance to tumor cells (Wu et al. 2018). Nowadays, there are two main anti-cancer mechanisms of nanocarriers commonly used in clinical practice. One is passive targeting, i.e., we strengthen the enhanced permeability and retention effect, so that nanocarriers are abundantly present at the tumor site, thereby extending the drug half-life and enhancing the anti-cancer efficiency. The other is active targeting, i.e., there are specific materials of identifying tumor tissues on its surface. To date, the applied advantages of active targeting nanocarriers in the treatment of cancers have still remained unclear. PLG-g-mPEG is a nanocarrier

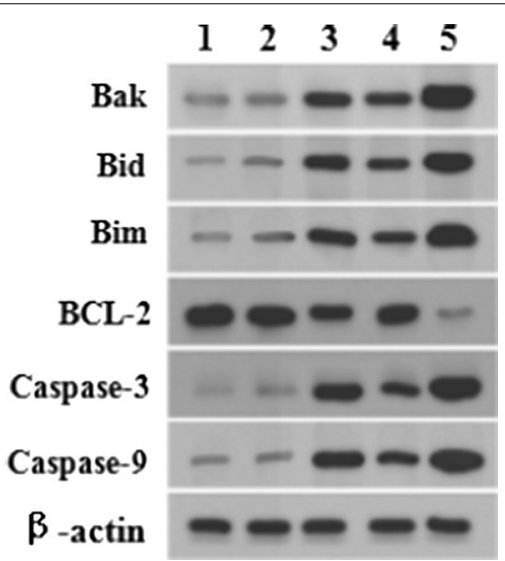

Fig. 6 The effect of CDDP/resiquimod/PLG-g-mPEG on the expressions of apoptosis-associated genes and proteins in SW626 cells. 1 for control group; 2 for CDDP group; 3 for resiquimod; 4 for PLG-g-mPEG group; 5 for CDDP/resiquimod/PLG-g-mPEG group 

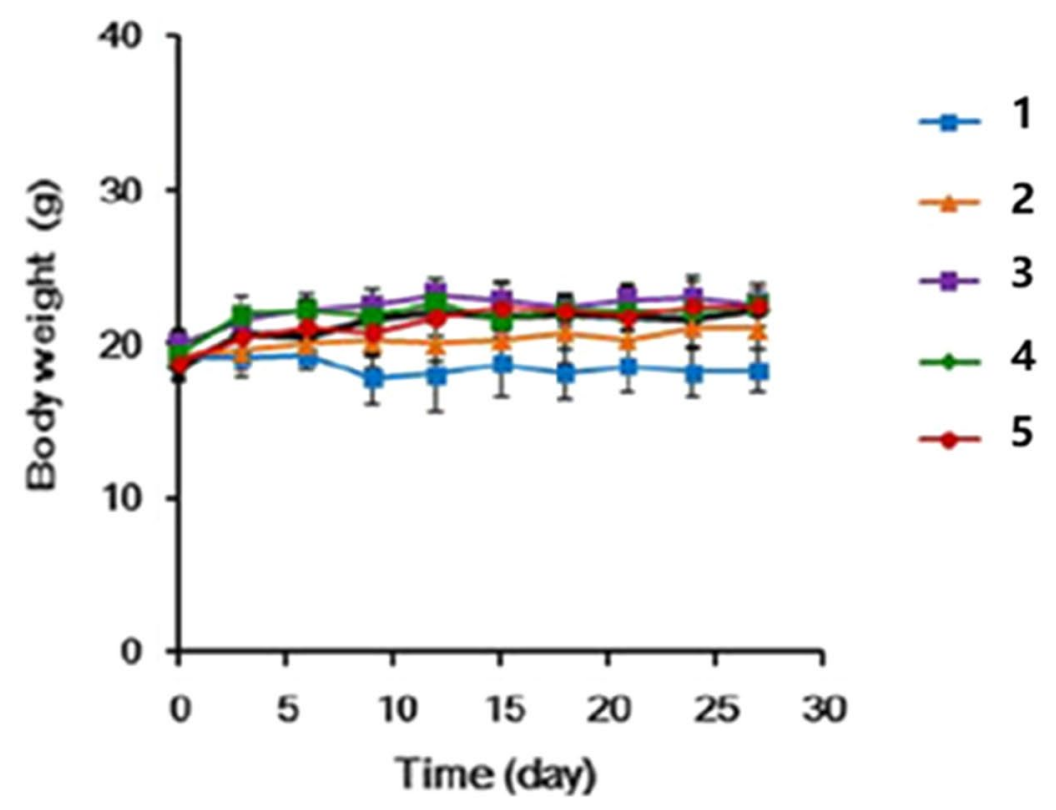

A
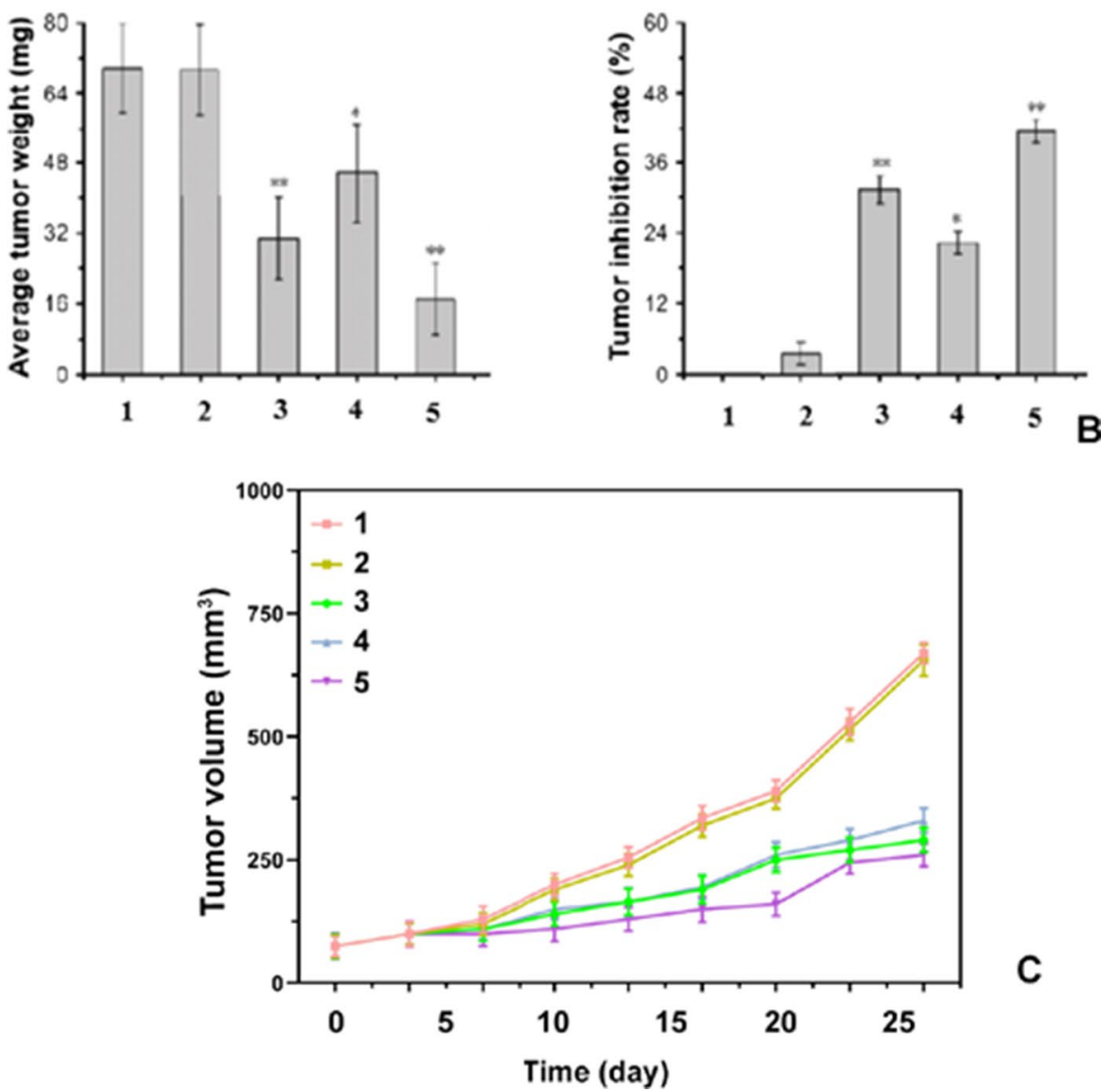

C

Fig. 7 The inhibitory effect of CDDP/resiquimod/PLG-g-mPEG on cancers in the boy. 1 for negative control group; 2 for resiquimod group; 3 for CDDP group; 4 for PLG-g-mPEG group; 5 for CDDP/resiquimod/ PLG-g-mPEG group; $\mathbf{A}$ for mouse weight change; $\mathbf{B}$ for the tumor weight and the inhibition rate of mice; $\mathbf{C}$ for tumor volume change 
consisting of rich in side chain carboxyl groups, and has been proven to have a stable passive targeting effect.

Previous studies have discovered that after PLG-g-mPEG is dissolved in water, its side chain carboxyl group can react with chlorine atoms in cisplatin to form nanomicelles, which significantly improves the pharmacokinetics and biodistribution of cisplatin, thereby enhancing the anti-cancer efficacy and reducing side effects (Yifei 2015). Besides, Yu and colleagues prepared a cisplatin-loaded PLG-g-mPEG nanoparticle via simple complexation of PLG-g-mPEG with cisplatin, and realized effective suppression of MCF-7 tumor growth (Yu et al. 2016). It is also reported that a multi-drug loaded PEG-based nanoparticle that contains resiquimod exhibited both chemo- and immunetherapy effect to ameliorate solid tumors resistant to first-line therapies (Silva et al. 2019). Basing on these previous reported studies and results from our experiments, we found that cisplatin and resiquimod could be directly loaded by PLG-g-mPEG in water phase, and then easily released in tumor sites to conduct therapeutic functions.

In recent years, it has been found that TAMs account for about $40 \%$ of tumor infiltrating cells and play a crucial role in the presence and development of ovarian cancer, being negatively correlated with the prognosis (He et al. 2017). TAMs are polarized into M1 phenotype and M2 phenotype. M1 possesses the tumor killing ability, whereas M2 promotes tumor growth. Studies have suggested that M2 can be induced into M1, which leads to a potent inhibition of the growth and metastasis of ovarian cancer (Zeng et al. 2019). Rodell et al. (2019) found that resiquimod retained the activity of macrophages polarization by activating TLR7/8, accelerated the transformation of M2 to M1 and effectively prevented tumor growth. In this study, PLG-g-mPEG was used to carry cisplatin and resiquimod. Our findings demonstrated that the LC and EE of CDDP/resiquimod/PLG-g-mPEG were $19.42 \%$ and $90.12 \%$, respectively, which met the ideal standard of nanodrug $(E E \geq 90 \%)$, with the average particle size of about $82.36 \mathrm{~nm}$ ranging from 5 to $200 \mathrm{~nm}$. For the time being, it was considered that the nanosystem demonstrated significant enhanced permeability and retention effect, which smoothly penetrated into tumor cells through tumor blood vessels. When zeta potential was $-23.69 \mathrm{mV}$, it indicated reduce in protein absorption and liver distribution (Huang et al. 2021), which was conducive to blood circulation, prolonged the residence of nanoparticles in the tumor site, and exerted a comparatively stronger anti-cancer effect. In the meantime, the results of flow cytometry and RT-PCR displayed that CDDP/resiquimod/PLG-g-mPEG was more beneficial to promote the transformation of M2-TAMs to M1 and reduce the polarization of M2-TAMs. Results from SRXRF imaging demonstrated notable internalization of CDDP/resiquimod/PLG-g-mPEG by tumor cells.

The results of in vitro experiments exhibited that PLG-g-mPEG had little impact on the growth of SW626 cells, and as the reaction time was prolonged, the cell survival rate was still maintained above $90 \%$, indicating that this nanocarrier was comparatively reliable. While comparing with the control group, we found that there was significantly reduce in the cell survival rate and plate clone formation rate of the CDDP/resiquimod/ PLG-g-mPEG group and CDDP, and as the reaction time was prolonged, the inhibitory effect of CDDP/resiquimod/PLG-g-mPEG on the growth and clone formation of SW626 cells raised. It was evident that CDDP/resiquimod/PLG-g-mPEG had an inhibitory effect on the growth and clone formation of tumor cells, but it was time-dependent. Moreover, 
PLG-g-mPEG was used to carry CDDP and resiquimod, which had a strong synergistic anti-cancer effect. From Western blotting, it was found that, compared against the control group, the relative expressions of Bak, Bid, Bim, Caspase-3 and Caspase-9 in the CDDP/resiquimod/PLG-g-mPEG group increased sharply, whereas the relative expression of BCL-2 decreased dramatically. It was suggested that CDDP/resiquimod/PLGg-mPEG not only inhibited the growth of SW626 cells, but also induced apoptosis to achieve the aim of anti-ovarian cancer, which was in line with previous results (Shengcai 2019). The outcomes of in vivo experiments showed that the weight of mice in each group fluctuated during medication, but there was no $\geq 20 \%$ weight loss or even death, indicating that the nanocarrier was comparatively reliable. What's more, we found that, compared with the negative control group, CDDP, PLG-g-mPEG and CDDP/resiquimod/PLG-g-mPEG markedly inhibited the growth of tumor cells instead of resiquimod, and CDDP/resiquimod/PLG-g-mPEG was the most effective drug for the inhibition of cancers. It has been further confirmed that CDDP/resiquimod/PLG-g-mPEG may be a new treatment for ovarian cancer.

\section{Conclusion}

In summary, using PLG-g-mPEG used to carry CDDP and resiquimod can effectively achieve the targeted delivery of chemotherapy and immunotherapy, with a strong synergistic anti-cancer effect.

Abbreviations

LC: Loading content; EE: Encapsulation efficiency; TAMs: Tumor-associated macrophages; PBS: Phosphate-buffered saline; $\mathrm{Cl}$ : Combination index.

\section{Acknowledgements}

Not applicable.

Authors' contributions

WY designed and performed experiments; SQ collected, analyzed data and wrote the paper. Both the authors read and approved the final manuscript.

Funding

Not applicable.

Availability of data and materials

The datasets used and analyzed during the current study are available from the corresponding author on reasonable request.

\section{Declarations}

Ethics approval and consent to participate

Not applicable.

\section{Consent for publication}

Not applicable.

Competing interests

The authors declare no competing financial interests.

Received: 29 April 2021 Accepted: 30 July 2021

Published online: 07 March 2022

References

Abouaitah K, Hassan HA, Swiderska-Sroda A et al (2020) Targeted nano-drug delivery of colchicine against colon cancer cells by means of mesoporous silica nanoparticles. Cancers 12(1):144-144 
Da Silva CG, Camps MGM, Li T et al (2019) Effective chemoimmunotherapy by co-delivery of doxorubicin and immune adjuvants in biodegradable nanoparticles. Theranostics 9(22):6485-6500

Davis KJ, Carrall JA, Lai B et al (2012) Does cytotoxicity of metallointercalators correlate with cellular uptake or DNA affinity? Dalton Trans 41(31):9417-9426

Figueiredo P, Lepland A, Scodeller P et al (2020) Peptide-guided resiquimod-loaded lignin nanoparticles convert tumor-associated macrophages from M2 to M1 phenotype for enhanced chemotherapy. Acta Biomater 7061 (20):30561-30564

Franken NA, Rodermond HM, Stap J et al (2006) Clonogenic assay of cells in vitro. Nat Protoc 1(5):2315-2319

Grabosch S, Bulatovic M, Zeng F et al (2019) Cisplatin-induced immune modulation in ovarian cancer mouse models with distinct inflammation profiles. Oncogene 38(13):2380-2393

$\mathrm{He} \mathrm{H}$, Chiu AC, Kanada M et al (2017) Imaging of tumor-associated macrophages in a transgenic mouse model of orthotopic ovarian cancer. Mol Imaging Biol 19(5):694-702

Huang D, Sun L, Huang L et al (2021) Nanodrug delivery systems modulate tumor vessels to increase the enhanced permeability and retention effect. J Pers Med 11(2):124-124

Jin EK, Jung-Yun L, Ji NE et al (2017) Long-term survival analysis of intraperitoneal versus intravenous chemotherapy for primary ovarian cancer and comparison between carboplatin- and cisplatin-based intraperitoneal chemotherapy. J Korean Med Sci 32(12):2021-2028

Le TN, Harvey RE, Kim CK et al (2017) A retrospective evaluation of activity of gemcitabine/platinum regimens in the treatment of recurrent ovarian cancer. Gynecol Oncol Res Pract 4(1):16-16

Li Y, Yang H, Yao J et al (2018) Glutathione-triggered dual release of doxorubicin and camptothecin for highly efficient synergistic anticancer therapy. Colloids Surf B Biointerfaces 169(38):273-279

Li H, Somiya M, Kuroda S (2021) Enhancing antibody-dependent cellular phagocytosis by re-education of tumor-associated macrophages with resiquimod-encapsulated liposomes. Biomaterials 268:120601

Lu R, Groer C, Kleindl PA et al (2019) Formulation and preclinical evaluation of a toll-like receptor 7/8 agonist as an antitumoral immunomodulator. J Control Release 306:165-176

Luo D, Qiong L, Xu M et al (2019) Incidence and mortality of ovarian cancer in Zhejiang cancer registration areas, 2010-2014. China Cancer 28(2):105-109

Michaelis KA, Norgard MA, Zhu X et al (2019) The TLR7/8 agonist R848 remodels tumor and host responses to promote survival in pancreatic cancer. Nat Commun 10(1):4682

O'Connor A, Brasher CJ, Slatter DA et al (2017) LipidFinder: a computational workflow for discovery of lipids identifies eicosanoid-phosphoinositides in platelets. JCl Insight 2(7):e91634

Pockros PJ, Guyader D, Patton H et al (2007) Oral resiquimod in chronic HCV infection: safety and efficacy in 2 placebocontrolled, double-blind phase lla studies. J Hepatol 47(2):174-182

Rodell CB, Arlauckas SP, Cuccarese MF et al (2018) TLR7/8-agonist-loaded nanoparticles promote the polarization of tumour-associated macrophages to enhance cancer immunotherapy. Nat Biomed Eng 2(8):578-588

Rodell CB, Ahmed MS, Garris CS, Pittet MJ, Weissleder R (2019) Development of adamantane-conjugated TLR7/8 agonists for supramolecular delivery and cancer immunotherapy. Theranostics 9(26):8426-8436

Savage P, Horton V, Moore J et al (1996) A phase I clinical trial of imiquimod, an oral interferon inducer, administered daily. Br J Cancer 74(9):1482-1486

Scholch S, Rauber C, Tietz A et al (2015) Radiotherapy combined with TLR7/8 activation induces strong immune responses against gastrointestinal tumors. Oncotarget 6(7):4663-4676

Shengcai Y (2019) Design of polymer nano drug delivery system and its anti-tumor research

Spiliotis J, lavazzo C, Fotiou A et al (2020) Upfront or intermediate treatment of advanced ovarian cancer patients with cytoreduction plus HIPEC: results of a retrospective study. J Surg Oncol 44(2):30-34

Thauvin C, Widmer J, Mottas I et al (2019) Development of resiquimod-loaded modified PLA-based nanoparticles for cancer immunotherapy: a kinetic study. Eur J Pharm Biopharm 139:253-261

Wu T, Huang H, Sheng Y et al (2018) Transglutaminase mediated PEGylation of nanobody for targeted nano-drug delivery. J Mater Chem B 6(7):1011-1017

Yifei L (2015) Applied research of Cisplatin-loaded nanoparticles in the treatment of osteosarcoma. Jilin University

Yin T, He S, Wang Y (2015) Toll-like receptor 7/8 agonist, R848, exhibits antitumoral effects in a breast cancer model. Mol Med Rep 12(3):3515-3520

Yu H, Tang Z, Li M, Song W et al (2016) Cisplatin loaded poly(L-glutamic acid)-g-methoxy poly(ethylene glycol) complex nanoparticles for potential cancer therapy: preparation, in vitro and in vivo evaluation. J Biomed Nanotechnol 12(1):69-78

Yu M, Zhang C et al (2019) Intratumoral injection of gels containing losartan microspheres and (PLG-g-mPEG)-cisplatin nanoparticles improves drug penetration retention and anti-tumor activity. Cancer Lett 442(48):396-408

Zeng XY, Xie H, Yuan J et al (2019) M2-like tumor-associated macrophages-secreted EGF promotes epithelial ovarian cancer metastasis via activating EGFR-ERK signaling and suppressing IncRNA LIMT expression. Cancer Biol Ther 20(7):1-11

\section{Publisher's Note}

Springer Nature remains neutral with regard to jurisdictional claims in published maps and institutional affiliations. 